\title{
A New Understanding on the Problem That the Quintic Equation Has No Radical Solutions
}

\author{
Xiaochun Mei \\ Department of Theoretical Physics and Pure Mathematics, Institute of Innovative Physics in Fuzhou, Fuzhou, China \\ Email: ycwlyjs@yeah.net
}

How to cite this paper: Mei, X.C. (2020) A New Understanding on the Problem That the Quintic Equation Has No Radical Solutions. Advances in Pure Mathematics, 10, 508-539.

https://doi.org/10.4236/apm.2020.109032

Received: August 6, 2020

Accepted: September 11, 2020

Published: September 14, 2020

Copyright (c) 2020 by author(s) and Scientific Research Publishing Inc. This work is licensed under the Creative Commons Attribution International License (CC BY 4.0).

http://creativecommons.org/licenses/by/4.0/

(c) (i) Open Access

\begin{abstract}
It is proved in this paper that Abel's and Galois's proofs that the quintic equations have no radical solutions are invalid. Due to Abel's and Galois's work about two hundred years ago, it was generally accepted that general quintic equations had no radical solutions. However, Tang Jianer et al. recently prove that there are radical solutions for some quintic equations with special forms. The theories of Abel and Galois cannot explain these results. On the other hand, Gauss et al. proved the fundamental theorem of algebra. The theorem declared that there were $n$ solutions for the $n$ degree equations, including the radical and non-radical solutions. The theories of Abel and Galois contradicted with the fundamental theorem of algebra. Due to the reasons above, the proofs of Abel and Galois should be re-examined and re-evaluated. The author carefully analyzed the Abel's original paper and found some serious mistakes. In order to prove that the general solution of algebraic equation he proposed was effective for the cubic equation, Abel took the known solutions of cubic equation as a premise to calculate the parameters of his equation. Therefore, Abel's proof is a logical circular argument and invalid. Besides, Abel confused the variables with the coefficients (constants) of algebraic equations. An expansion with 14 terms was written as 7 terms, 7 terms were missing. We prefer to consider Galois's theory as a hypothesis rather than a proof. Based on that permutation group $S_{5}$ had no true normal subgroup, Galois concluded that the quintic equations had no radical solutions, but these two problems had no inevitable logic connection actually. In order to prove the effectiveness of radical extension group of automorphism mapping for the cubic and quartic equations, in the Galois's theory, some algebraic relations among the roots of equations were used to replace the root itself. This violated the original definition of automorphism mapping group, led to the confusion of concepts and arbitrariness. For the general cubic and quartic algebraic equations, the actual solving processes do not satisfy the tower structure of Galois's solvable group. The resolvents of cubic and quartic equations
\end{abstract}


are proved to have no symmetries of Galois's soluble group actually. It is invalid to use the solvable group theory to judge whether the high degree equation has a radical solution. The conclusion of this paper is that there is only the $S_{n}$ symmetry for the $n$ degree algebraic equations. The symmetry of Galois's solvable group does not exist. Mathematicians should get rid of the constraints of Abel and Galois's theories, keep looking for the radical solutions of high degree equations.

\section{Keywords}

Quintic Equation, Gauss Basic Theorem of Algebra, Radical Solution, Abel's Theory, Galois's Theory, Solvable Group, Lagrange's Resolvents

\section{Introduction}

The so-called radical solution problem of quintic equation is to find a general formula to express the roots of arbitrary quintic equation in the radical forms of equation's coefficients uniformly.

People knew how to solve quadratic equations in the seventh century. Through the hard works of mathematicians, the general solutions of cubic and quartic equations were found about 450 years ago. But the general solutions of quintic equation and higher degree equations (called as high degree equations below) are still unknown at present, though some special solutions have been obtained for some quintic equations with special forms.

In the history of mathematics, Euler, d'Alembert, Lagrange, and Guass et al. tried to solve quintic equation but all of them failed. Until 1826, Abel published a paper to prove that general quintic equation cannot be solved [1]. Then Galois used the theory of group to prove the same result by proposing a judgment ruler for the solvability of general algebraic equations [2]. Galois's proof was regarded as more general and strict. After that, this problem was regarded to be perfectly solved. Most mathematicians stopped thinking about it.

Abel's paper proved that the general quintic equation had no solution, without saying that there were no radical solutions. Galois introduced the concept of radical expansion of domain and turned the problem into that the quintic equation had no radical solutions. There are subtle differences here, but Galois's saying often leads to misunderstandings. The absence of radical solutions for the quintic equation is a technical but narrow saying. More accurate one is that we cannot find a general formula to describe the solutions of a general quintic equation uniformly.

On the other hand, according to the fundamental theorem of algebra, there were $n$ solutions for any $n$ degree equation with one variable. It indicates that any $n$ degree equation can always be written as

$$
\begin{aligned}
f(x) & =a_{n} x^{n}+a_{n-1} x^{n-1}+a_{n-2} x^{n-2}+\cdots+a_{1} x+a_{0} \\
& =\left(x-x_{1}\right)\left(x-x_{2}\right)\left(x-x_{3}\right) \cdots\left(x-x_{n}\right)=0
\end{aligned}
$$


Here $a_{1}, a_{2}, \cdots, a_{n} \in Q$ are the rational numbers and $x_{1}, x_{2}, \cdots, x_{n}$ are the roots of the equation. If we add a constrain condition, suppose that Equation (1) is the so-called irreducible on $Q$, i.e., the equation has no the solutions of rational numbers, according to the fundamental theorem of algebra, it still has the solutions of irrational numbers or complex numbers.

However, according to the theories of Able and Galois, if $f(x)$ is irreducible on $Q$, it means that the solutions $x_{1}, x_{2}, \cdots, x_{n}$ cannot be expressed by the radical sign forms of coefficient $a_{1}, a_{2}, \cdots, a_{n}$, i.e., $x_{i}$ cannot be written in the forms of $\sqrt{a_{k}+\sqrt{a_{j}}}$ and $\sqrt[5]{a_{i}+\sqrt{a_{j}}+\sqrt[3]{a_{k}}}$ and so on. Because the absolute value of a complex number $z=a+i b=|z| \mathrm{e}^{i \theta}$ can be expressed in the form of radical sign with $|z|=\sqrt{a^{2}+b^{2}}$, there are no radical solutions means that the possibility of complex number's solutions is excluded for the quintic equations (if $|z|$ is an irrational number).

So the assertion that high degree equation has no radical solution is strange. If its solution cannot be expressed in the radical forms, what can we use to describe the solutions? As we known that number fields can be divided into rational number, irrational number, complex number and transcendental number. So-called having no radical solutions means that the roots of equations cannot be irrational numbers. Because we have defined that $f(x)$ is irreducible on $Q$, the solutions of equations cannot be rational numbers. Because the transcendental numbers cannot be the roots of algebraic equations, what remained are complex numbers without containing radical signs.

On the other hand, it has been proved that the complex number roots of algebraic equations are conjugated. An odd order polynomial equation has a real number root at least [3]. The quintic equation has four imaginary number roots and one real number root, or two imaginary roots and three real roots. If these real roots are neither rational numbers nor irrational numbers or transcendental numbers, what are they?

It is obvious that the theory of Abel and Galois contradicts with the basic theorem of algebra. There must be one which is wrong. Gauss himself even proposed three or four methods to prove the basic theorem of algebra. After Gauss, many mathematician researched this problem. More than one hundred proofs were proposed up to now [4]. It notes that the basic theorem of algebra should be reliable, so the judgment that high degree equations have no radical solutions is doubtful.

Since the turn of this century, some progress has been made in the study of solving quintic equations. Professor Tang Jianer in Mathematics Department, Shanghai Finance and Economics University published a paper in 2012 to prove that same special quintic equations had radical solutions [5]. Other persons also made the same work [6] [7]. Zheng Liangfei solved a lot of quintic equations with number coefficients by using same special methods [8]. All of these solutions cannot be explained by the theories of Abel and Galois. Considering that the solution of quintic equation is one of the most fundamental problems in al- 
gebra, it is necessary to re-examine Abel and Galois's proofs.

The research in this paper finds that Abel's proof does not hold. His calculations contained logic confusion and basic conceptual mistake, which made Abel's paper hard to be understood. The general form of algebra equation's solution proposed by Abel is inconsistent with the practical forms of cubic and quartic equation's solutions.

Though the group theory of Galois was a great discovery, Galois's theory of solvable group had not solved the problem of high degree equation's solutions. We prefer to consider it as a hypothesis than to be a proof. Based on that the permutation group $S_{5}$ of quintic equation was a single group without true normal subgroup, Galois declared that the quintic equation had no radical solutions. Galois's deduction is ill-founded because both have no inevitable logic relation. The theory of radical extension did not match with the practical process of solving cubic and quartic equations too.

The main points of more detailed content are as follows.

1) To introduce the calculation method of Tang Jianer, cite his two radical solutions of special quintic equations. For example, when $a_{5}=1, a_{4}=a_{2}=0$ and $a_{3}^{2}=5 a_{1}$ in Equation (1), the solution of quintic equation had the similar structure of cubic equation. These five equations calculated by Tang Jianer satisfy permutation group $\mathcal{S}_{5}$ without true normal subgroups. There are no Galloi's solvable groups for them, but they still have radical solutions.

2) Abel's proof is analyzed carefully and some serious mistakes are founded. In order to prove that the solution form of general algebraic equation he proposed was valid for the cubic equation, Abel took the known solution of cubic equation as the premise to calculate the parameters of his equation. So Abel's proof is a circular argument without meaning.

3) Abel confused the concepts of variables with the coefficients (constants) of algebraic equations, violated the basic principle of solving equation. An expansion with 14 terms was written into 7 items, 7 items were missed. It is proved that the solutions of cubic and quartic equation do not satisfy the form given by Abel, and there is no reason to think that the solutions of higher order equation can satisfy the Abel's form.

4) Galois's solvable group theory is far-fetched and its conclusion and premise are contradictory. The Galois's theory admitted that the symmetries between the roots and the coefficients of quinic equations existed and used the permutation group $S_{5}$ to describe the symmetries. Due to that the $S_{5}$ group had no true normal subgroup, the quintic equation was regarded to have no radical solution. However, such an argument is not well-founded. Since it has assumed that the symmetry of the roots and coefficients can be described by the permutation group $S_{5}$, it is actually accepted the existence of equation's solution. It just doesn't have the symmetry of true normal subgroup.

5) The automorphism mapping concept of radical extension was used to construct the Galois group. The function of an automorphism mapping group operator is to change the roots of an algebraic equation into other roots of the same 
equation, or to keep the roots themselves unchanged.

However, in order to prove the effectiveness of this method for the cubic and quartic equations, Galois's theory actually used the algebraic relations of same roots to replace roots themselves. This is not only a substitution of concepts, but also introduces arbitrariness, results in the destruction of uniqueness.

6) The theory of radical extension did not match with the practical process. The practical process of solving an equation did not meet with the tower structure of Galois's group by adding the multiple roots layer by layer. It obeys the ruler of decreasing degree of $S_{n}$ group. When we find a solution for $n$ degree equation, the degree of equation is decreased to $n-1$. The $S_{n}$ symmetry between the roots and coefficients are decreased to $S_{n-1}$. This process has nothing to do with the resolvable group with tower structure. The theory is only something formal.

7) It is proved that the resolvents of general cubic and quartic equations can only partially satisfy the symmetries of solvable groups, but cannot satisfy all the symmetries. For example, the resolvent of cubic equation can keep unchanged under the permutation (123) of normal subgroup $A_{3}$ of $S_{3}$ group, but cannot keep unchanged under the permutation (132).

In sum, the basic theorem of algebra has proved that the general solutions of high degree equations exist. Tang Jianer et al. had found several radical solutions for some special quintic and sixtic equations. The Galois's theory cannot explain why they may be the exceptions of Galois resolvable group. By considering the results reveled in this paper that there is only $S_{n}$ symmetry between the solutions and coefficients for the $\mathrm{n}$ degree algebraic equations, there is no the symmetry of Galois's solvable group, the traditional conclusion that high degree equations have no radical solutions should be given up. Mathematicians should get rid of the constraints of Abel and Galois's theories, keep to looking for the radical solutions of general high degree equations.

\section{The Radical Solutions of Same Special Quintic Equations}

Sheng Xinping earliest proposed the matrix method to solve the quartic equation [7]. Fang Jun and Kong Zhihui used the similar method to solve three kinds of special sextic equations by reducing the equations to cubic equations [4]. But this method cannot be used to solve the quintic equations.

Based on this method, Tang Jianer used five variables to replace one variable and obtained the radical solutions of several special quintic equations. This method has general significance [5]. The quintic equation was written in the standard form with

$$
x^{5}+p x^{3}+q x^{2}+r x+s=0
$$

The solutions of quintic cyclotomic equation $\zeta^{5}-1=0$ are

$$
\zeta=\mathrm{e}^{i 2 \pi / 5}=\cos \frac{2 \pi}{5}+i \sin \frac{2 \pi}{5}
$$

By introducing four variables $y, z, u, v$ and using the fifth degree circular de- 
terminant, Tang Jianer proved following identical equation

$$
x^{5}+p x^{3}+q x^{2}+r x+s=\prod_{j=0}^{4}\left(x+\zeta^{j} y+\zeta^{2 j} z+\zeta^{3 j} u+\zeta^{4 j} v\right)
$$

The relations between coefficients $p, q, r, s$ and variables $y, z, u, v$ are [5]

$$
\begin{gathered}
p=-5(y v+z u) \\
q=5\left(y z^{2}+z v^{2}+u y^{2}+v u^{2}\right) \\
r=5\left(y v^{2}+z^{2} u^{2}-y^{3} z-z^{3} v-u^{3} y-v^{3} u-y z u v\right) \\
s=y^{5}+z^{5}+u^{5}+v^{5}-5 y^{3} u v-5 z^{3} y u-5 u^{3} z v \\
-5 v^{3} y z+5 u^{2} v^{2} y+5 u^{2} v^{2} z+5 z^{2} v^{2} u+5 y^{2} z^{2} v
\end{gathered}
$$

According to Equation (2), Equation (4) indicates

$$
x+\zeta^{j} y+\zeta^{2 j} z+\zeta^{3 j} u+\zeta^{4 j} v=0
$$

The five solutions of quintic equations are

$$
x_{j}=-\zeta^{j} y-\zeta^{2 j} z-\zeta^{3 j} u-\zeta^{4 j} v, j=0,1,2,3,4
$$

If we can use $p, q, r, s$ to represent $y, z, u, v$ based on Equations (5)-(8), the five solutions of quintic equations are obtained. Tang Jianer provided five special examples in his paper. Here we quote two of them.

1) Let $q=0$ in Equation (2), the equation becomes

$$
x^{5}+p x^{3}+r x+s=0
$$

According to Equation (6), for $q=0$, we let $y=0$ and $v=0$. Substituting them in Equations (5), (7) and (8), we get

$$
\begin{gathered}
p=-5 z u \\
r=5 z^{2} u^{2} \\
s=u^{5}+z^{5}
\end{gathered}
$$

From Equations (12) and (13), we get $p^{2}=5 r$. Form Equation (12), we have $z=-p /(5 u)$. Substituting them in Equation (14), we obtain

$$
u^{10}-s u^{5}-\left(\frac{p}{5}\right)^{5}=0
$$

The solution of Equation (15) is

$$
u^{5}=\frac{s}{2} \pm \sqrt{\left(\frac{s}{2}\right)^{2}+\left(\frac{p}{5}\right)^{5}}
$$

Take

$$
u=\left[\frac{s}{2}+\sqrt{\left(\frac{s}{2}\right)^{2}+\left(\frac{p}{5}\right)^{5}}\right]^{\frac{1}{5}}
$$

According to Equation (12), we get

$$
z=\left[\frac{s}{2}-\sqrt{\left(\frac{s}{2}\right)^{2}+\left(\frac{p}{5}\right)^{5}}\right]^{-\frac{1}{5}}
$$


Therefore, under the condition that Equation (15) is satisfied, the solution $(j=0)$ in Equation (10) is $x_{0}=-z-u$, or

$$
x_{0}=-\left[\frac{s}{2}+\sqrt{\left(\frac{s}{2}\right)^{2}+\left(\frac{p}{5}\right)^{5}}\right]^{\frac{1}{5}}-\left[\frac{s}{2}-\sqrt{\left(\frac{s}{2}\right)^{2}+\left(\frac{p}{5}\right)^{5}}\right]^{\frac{1}{5}}
$$

By considering Equation (14), it can be verified directly that Equation (19) is the solution of Equation (11). Another four solutions are determined by Equation (10). On the other side, for the cubic equation, we have

$$
\begin{gathered}
x^{3}+p x+s=0 \\
x_{0}=\left[-\frac{s}{2}+\sqrt{\left(\frac{s}{2}\right)^{2}+\left(\frac{p}{3}\right)^{3}}\right]^{\frac{1}{3}}+\left[-\frac{s}{2}-\sqrt{\left(\frac{s}{2}\right)^{2}+\left(\frac{p}{3}\right)^{3}}\right]^{\frac{1}{3}}
\end{gathered}
$$

It is interesting that the solution of quintic equation (11) is similar to that of the cubic equation (20) if the coefficients $p$ and $r$ in the equation satisfies the relation $p^{2}=5 r$.

2) Let $p=0$ in Equation (2), the equation becomes

$$
x^{5}+q x^{2}+r x+s=0
$$

Take $u=v=0$ in Equation (5), substituting them in Equations (6)-(8), we get

$$
\begin{gathered}
q=5 y z^{2} \text { or } y z^{2}=\frac{1}{5} q \\
r=-5 y^{3} z \text { or } y^{3} z=-\frac{1}{5} r \\
s=y^{5}+z^{5}
\end{gathered}
$$

Theses three formulas above satisfy

$$
\frac{\left(y^{3} z\right)^{2}}{y z^{2}}+\frac{\left(y z^{2}\right)^{3}}{y^{3} z}=y^{5}+z^{5}
$$

By considering Equations (23)-(25), Equation (26) can be written as

$$
q^{4}+25 q r s-5 r^{3}=0
$$

From Equations (23) and (24), we have

$$
y=\left(\frac{r^{2}}{5 q}\right)^{1 / 5}, z=-\left(\frac{q^{3}}{25 r}\right)^{1 / 5}
$$

Therefore, one solution of Equation (22) is

$$
x_{0}=y+z=\left(\frac{r^{2}}{5 q}\right)^{1 / 5}-\left(\frac{q^{3}}{25 r}\right)^{1 / 5}
$$

Another four solutions are determined by Equation (10). We can use a concrete example to verify the solution. For the equation

$$
x^{5}+10 x^{2}+5 x-15 / 2=0
$$

We have $p=0, q=10, r=5, s=-15 / 2$. They satisfy Equation (26). Ac- 
cording to Equation (28), we get $y=2^{1 / 5}$ and $z=-8^{1 / 5}$. According to Equation (29), we have $x_{0}=2^{1 / 5}-8^{1 / 5} \approx 0.6451$. It can be verified directly that this value satisfies the equation (30).

It notes that the solutions (19) and (29) are described by the forms of radical signs. Thought they are obtained in special situations, the theories of Able and Galois cannot explain why the equations have the solution under these conditions. So the conclusion that quintic equations have no radical solutions does not hold.

\section{Brief Introduce to the Abel's Proof}

\subsection{Abel's Proof in $\mathbf{1 8 2 4}$}

The content in this chapter is cited from the first part of the Abel's original paper in 1824 and the paper published in 1826. The existing problems are discussed in Chapter 4. In the first part of 1824's paper, Abel wrote quintic equation as [1]

$$
y^{5}-a y^{4}+b y^{3}-c y^{2}+d y-e=0
$$

Then he assumed that the solution of Equation (31) would be in following form

$$
y=p+p_{1} R^{1 / m}+p_{2} R^{2 / m}+\cdots+p_{m-1} R^{(m-1) / m}
$$

Here $m$ was a primer and $R, p, p_{1}, p_{2}, \cdots$ had the same form as $y$. Continuously by this method, until they were represented as rational function of $a, b, c, d, e$.

By using $R / p_{1}^{m}$ to replace $R$, or taking $p_{1}=1$, Equation (32) could be written as

$$
y=p+R^{1 / m}+p_{2} R^{2 / m}+\cdots+p_{m-1} R^{(m-1) / m}
$$

Substituting Equation (33) in Equation (31), Abel obtained [1]

$$
q+q_{1} R^{1 / m}+q_{2} R^{2 / m}+\cdots+q_{m-1} R^{(m-1) / m}=0
$$

Here $q, q_{1}, q_{2}, \cdots, q_{m-1}$ are the rational functions of $a, b, c, d, e$ and $p, q_{2}, \cdots$ as well as $R$. Then Abel proved by reduction to absurdity that in order to satisfy Equation (34), it must have

$$
q=q_{1}=q_{2}=\cdots=q_{m-1}=0
$$

The proof is below. Let $z=R^{1 / m}$ and consider Equation (34), two equations could be obtained

$$
\begin{gathered}
z^{m}-R=0 \\
q+q_{1} z+q_{2} z^{2}+\cdots+q_{m-1} z^{m-1}=0
\end{gathered}
$$

If $q, q_{1}, q_{2}, \cdots, q_{m-1}$ did not equal to zeros simultaneously, Equations (36) and (37) must have one or more than one common root. If the number of roots was $k$, we could find an equation with degree $k$. Its parameters were the rational function of $R, q, q_{1}, q_{2}, \cdots, q_{m-1}$. Supposed the equation was 


$$
r+r_{1} z+r_{2} z^{2}+\cdots+r_{k} z^{k}=0
$$

Its all roots were the same with the roots of Equation (35). Because the root of this equation had the form $\alpha_{\mu} z$, here $\alpha_{\mu}$ was one root of equation $\alpha_{\mu}^{m}-1=0$. Through the replace, following $k$ equations could be obtained

$$
\begin{aligned}
& r+r_{1} z+r_{2} z^{2}+\cdots+r_{k} z^{k}=0 \\
& r+\alpha r_{1} z+\alpha^{2} r_{2} z^{2}+\cdots+\alpha^{k} r_{k} z^{k}=0 \\
& \vdots \\
& r+\alpha_{k-1} r_{1} z+\alpha_{k-2}^{2} r_{2} z^{2}+\cdots+\alpha_{k-1}^{k} r_{k} z^{k}=0
\end{aligned}
$$

From these $k$ equations, the value of rational function $z$ could be obtained which were be represented by $r, r_{1}, r_{2}, \cdots, r_{k}$. Because these quantities themselves were the rational functions of $a, b, c, d, e, R$ and $p, p_{1}, p_{2}, \cdots$, so $z$ is the rational function of those quantities.

However, according to the definition $z=R^{1 / m}, Z$ was generally impossible to be a rational number, thus contradiction was caused. To make Equation (37) true, only the result was Equation (35). As for what was the relation between Equation (35) and Equation (33), Abel's paper in 1824 had not provided any explanation.

Then, let $y_{1}, y_{2}, y_{3}, \cdots, y_{m}$ represent the roots of $m$ degree equation, $\alpha$ be the root of the equation $x^{m-1}+x^{m-2}+\cdots+x+1=0$, Abel assumed

$$
\begin{gathered}
y_{1}=p+R^{1 / m}+p_{2} R^{2 / m}+\cdots+p_{m-1} z^{(m-1) / m} \\
y_{2}=p+\alpha R^{1 / m}+\alpha^{2} p_{2} R^{2 / m}+\cdots+\alpha^{m-1} p_{m-1} z^{(m-1) / m} \\
\vdots \\
y_{m}=p+\alpha^{m-1} R^{1 / m}+\alpha^{m-2} p_{2} R^{2 / m}+\cdots+\alpha p_{m-1} z^{(m-1) / m}
\end{gathered}
$$

Based on Equation (40), by considering the exchange symmetry of different solutions, as well as the result of a paper published by Cauchy in Journal de l'ecole polytechnic que [9], Abel declared that $m$ could not be equal or greater than 5. In this way, Abel declared that the quintic equations had no solutions.

\subsection{Abel's Supplement Proof in $\mathbf{1 8 2 6}$}

In Abel's paper in 1824, no concrete calculation was taken to prove the effectiveness of formulas (33)-(35). In his paper in 1826, Abel made the calculation to prove Equations (33)-(35). The proof is below [10].

For the cubic equation, take $c=0$ for simplification, Equation (31) became

$$
y^{3}+d y-e=0
$$

Let $m=3$, Equation (33) became

$$
y=p+R^{1 / 3}+p_{2} R^{2 / 3}
$$

Then Able proved that for Equation (41), one could take $p=0$ in Equation (42). The three solutions of Equation (41) are

$$
y_{1}=R_{1}^{1 / 3}+R_{2}^{1 / 3}=\left[\frac{e}{2}+\sqrt{\left(\frac{e}{2}\right)^{2}+\left(\frac{d}{3}\right)^{3}}\right]^{\frac{1}{3}}+\left[\frac{e}{2}-\sqrt{\left(\frac{e}{2}\right)^{2}+\left(\frac{d}{3}\right)^{3}}\right]^{\frac{1}{3}}
$$




$$
\begin{aligned}
& y_{2}=\omega R_{1}^{1 / 3}+\omega^{2} R_{2}^{1 / 3}=\omega\left[\frac{e}{2}+\sqrt{\left(\frac{e}{2}\right)^{2}+\left(\frac{d}{3}\right)^{3}}\right]^{\frac{1}{3}}+\omega^{2}\left[\frac{e}{2}-\sqrt{\left(\frac{e}{2}\right)^{2}+\left(\frac{d}{3}\right)^{3}}\right]^{\frac{1}{3}} \\
& y_{3}=\omega^{2} R_{1}^{1 / 3}+\omega R_{2}^{1 / 3}=\omega^{2}\left[\frac{e}{2}+\sqrt{\left(\frac{e}{2}\right)^{2}+\left(\frac{d}{3}\right)^{3}}\right]^{\frac{1}{3}}+\omega\left[\frac{e}{2}-\sqrt{\left(\frac{e}{2}\right)^{2}+\left(\frac{d}{3}\right)^{3}}\right]^{\frac{1}{3}}
\end{aligned}
$$

Here $R_{1} \neq R_{2}, \omega$ is the root of equation $y^{3}-1=0$ with the relation $1+\omega+\omega^{2}=0$. By adding Equations (43)-(45) together, according to the Vieta formula, one gets

$$
\begin{aligned}
& \left(y_{1}+y_{2}+y_{3}\right) \\
& =\left(1+\omega+\omega^{2}\right)\left[\frac{e}{2}+\sqrt{\left(\frac{e}{2}\right)^{2}+\left(\frac{d}{3}\right)^{3}}\right]^{\frac{1}{3}}+\left(1+\omega+\omega^{2}\right)\left[\frac{e}{2}-\sqrt{\left(\frac{e}{2}\right)^{2}+\left(\frac{d}{3}\right)^{3}}\right]^{\frac{1}{3}} \\
& =0
\end{aligned}
$$

Therefore, for Equation (42), Abel thought that one also had

$$
y_{1}+y_{2}+y_{3}=3 p+\left(1+\omega+\omega^{2}\right) R^{1 / 3}+\left(1+\omega+\omega^{2}\right) p_{2} R^{2 / 3}=3 p=0
$$

It also indicated $p=0$, so Equation (42) became

$$
y=R^{1 / 3}+p_{2} R^{2 / 3}
$$

Substituting Equation (48) in Equation (41) and witting it in the form of Equation (34), Abel got

$$
\left(R+p_{2}^{3} R^{2}-e\right)+\left(3 p_{2} R+d\right) R^{1 / 3}+\left(3 p_{2}^{2} R+d p_{2}\right) R^{2 / 3}=0
$$

Or writing Equation (49) as

$$
q+q_{1} R^{1 / 3}+q_{2} R^{2 / 3}=0
$$

According to Equation (35), the results should be

$$
\begin{gathered}
q=R+p_{2}^{3} R^{2}-e=0 \\
q_{1}=3 p_{2} R+d=0 \\
q_{2}=3 p_{2}^{2} R+d p_{2}=0
\end{gathered}
$$

One gets $p_{2}=-d /(3 R)$ from Equation (52). Substituting it in Equation (53), the right side was just equal to zero. Substitute $p_{2}=-d /(3 R)$ in Equation (51), the result is

$$
R^{2}-e R-(d / 3)^{3}=0
$$

This is the second degree equation with two solutions

$$
\begin{aligned}
& R_{+}=\frac{e}{2}+\sqrt{\left(\frac{e}{2}\right)^{2}+\left(\frac{d}{3}\right)^{3}} \\
& R_{-}=\frac{e}{2}-\sqrt{\left(\frac{e}{2}\right)^{2}+\left(\frac{d}{3}\right)^{3}}
\end{aligned}
$$

There is the relation 


$$
p_{2} R_{+}^{2 / 3}=-\frac{d}{3 R_{+}} R_{+}^{2 / 3}=-\frac{d}{3} R_{+}^{-1 / 3}
$$

The Vieta's formula of Equation (54) is

$$
R_{+}+R_{-}=e, R_{+} R_{-}=-(d / 3)^{3}
$$

By considering Equations (56) and (57), Equation (48) can be written as

$$
y=R_{+}^{1 / 3}+p_{2} R_{+}^{2 / 3}=R_{+}^{1 / 3}-\frac{d}{3} R_{+}^{-1 / 3}=R_{+}^{1 / 3}+R_{-}^{-1 / 3}
$$

Equations (58) and (43) had the same forms for $R_{+} \rightarrow R_{1}$ and $R_{-} \rightarrow R_{2}$, so Abel thought that his basic Formula (33) of general algebraic equation's solution was valid for the cubic equation.

\section{The Problems Existing in Abel's Proof}

In the Abel's proof, the concepts were unclear and logical was confused. The calculations had some serious mistake. For the quadratic equation, Equation (35) does not hold in general. For the cubic and quartic equations, Equation (33) is untenable. Equation (35) is even less likely to be true. So Equation (40) cannot hold too.

1) For the quadratic equation, Equation (31) becomes

$$
y^{2}-d y+e=0
$$

Let $m=2$, according to Equation (33), there are two results

$$
y=p+R^{1 / 2}
$$

and

$$
y=p+R^{1 / 2}+p_{2} R+p_{1} R^{1 / 2}=p+\left(1+p_{1}\right) R^{1 / 2}+p_{2} R
$$

By solving Equation (59) directly, the result is

$$
y=\frac{d}{2} \pm \frac{\sqrt{d^{2}-4 e}}{2}=p \pm R^{1 / 2}
$$

where

$$
p=d / 2, \quad R=\left(d^{2}-4 e\right) / 4
$$

It is obvious that Equation (60) is tenable but Equation (61) does not hold. We only need to discuss Equation (60). Substituting Equation (60) in Equation (59), we get

$$
q+q_{1} R^{1 / 2}+q_{2} R=0
$$

where

$$
q=p^{2}-d p+e, q_{1}=2 p-d, q_{2}=1
$$

Substituting $p=d / 2$ in Equation (65), we get

$$
q=-d^{2} / 4+e, q_{1}=0, q_{2}=1
$$

According to Equation (35), Abel thought $q=q_{1}=q_{2}=0$, but it is obviously impossible. We have $q_{2} \neq 0$ certainly. Because the coefficients $d$ and $e$ are independent, we may have $d \neq 2 \sqrt{e}$, so we have $q \neq 0$ in general. For the quadratic equation, Abel's deduction result in Equation (35) is invalid. 
2) For the cubic equation, the formulas (51)-(53) are wrong. Equation (49) can be developed into 7 items

$$
-e+d R^{1 / 3}+d p_{2} R^{2 / 3}+R+3 p_{2} R^{4 / 3}+3 p_{2}^{2} R^{5 / 3}+p_{2}^{3} R^{2}=0
$$

But substituting Equation (42) in Equation (41), what we obtain is

$$
q^{\prime}+q_{1}^{\prime} R^{1 / 3}+q_{2}^{\prime} R^{2 / 3}+q_{3}^{\prime} R+q_{4}^{\prime} R^{4 / 3}+q_{5}^{\prime} R^{5 / 3}+q_{6}^{\prime} R^{2}=0
$$

where

$$
\begin{aligned}
& q^{\prime}=p^{3}+d p-e, \quad q_{1}^{\prime}=3 p^{2}+d, \quad q_{2}^{\prime}=3 p+3 p^{2} p_{2}+d p_{2}, \\
& q_{3}^{\prime}=1+6 p p_{2}, \quad q_{4}^{\prime}=3 p_{2}+3 p p_{2}^{2}, \quad q_{5}^{\prime}=3 p_{2}^{2}, \quad q_{6}^{\prime}=p_{2}^{3}
\end{aligned}
$$

Therefore, Equation (68) has 14 items. But in the Abel's calculation, Equations (49) or (67) only have 7 items, the other 7 items containing $p$ were missing. To write Equation (68) in the form of Equation (43), what we obtain is

$$
\left(q^{\prime}+q_{3}^{\prime} R+q_{6}^{\prime} R^{2}\right)+\left(q_{1}^{\prime}+q_{4}^{\prime} R\right) R^{1 / 3}+\left(q_{2}^{\prime}+q_{5}^{\prime} R\right) R^{2 / 3}=0
$$

According to Equation (35), three equations are obtained

$$
\begin{gathered}
q=q^{\prime}+q_{3}^{\prime} R+q_{6}^{\prime} R^{2}=p^{3}+d p-e+\left(3 p^{2}+d\right) R+p_{2}^{3} R^{2}=0 \\
q_{1}=q_{1}^{\prime}+q_{4}^{\prime} R=3 p^{2}+d+3 p_{2}\left(1+p p_{2}\right) R=0 \\
q_{2}=q_{2}^{\prime}+q_{5}^{\prime} R=3 p+3 p^{2} p_{2}+d p_{2}+3 p_{2}^{2} R=0
\end{gathered}
$$

Equations (71), (72) and (73) are a set of ternary cubic equations about three unknown invariables, of which form is more complex than Equation (41). Suppose we can solve this set of equations, the solutions are $R=R(d, e), \quad p=p(d, e)$ and $p_{2}=p_{2}(d, e)$. Because the parameters $d$ and $e$ are arbitrary, in general, we have $p(d, e) \neq 0$. So we also have $p_{2} \neq-d /(3 R)$ and cannot get Equation (54) and (55).

In fact, in general situation, we have $q \neq 0, q_{1} \neq 0, q_{2} \neq 0$. We have no reason to think that $q, q_{1}$ and $q_{2}$ should be equal to zero. Abel's proof of Equation (35) does not hold for cubic equation. It can be seen that Abel's proof for Equation (47) is a logical circular argument. He took what he want to prove as a premise. Equation (47) means that there are following relations.

$$
\begin{gathered}
y_{1}=p+R^{1 / 3}+p_{2} R^{2 / 3} \\
y_{2}=p+\omega R^{1 / 3}+\omega^{2} p_{2} R^{2 / 3} \\
y_{3}=p+\omega^{2} R^{1 / 3}+\omega p_{2} R^{2 / 3}
\end{gathered}
$$

By adding Equations (74)-(76) together, Equation (47) is obtained. However, Abel needed to prove in advance that (74), (75) and (76) were the solutions of cubic equation, but he had not done it. What he did was to make Equations (43) and (48) to be equal with

$$
p+R^{1 / 3}+p_{2} R^{2 / 3}=R_{1}^{1 / 3}+R_{2}^{1 / 3}
$$

Then took $R^{1 / 3}=R_{1}^{1 / 3}$ and $p_{2} R^{2 / 3}=R_{2}^{1 / 3}$ directly and obtained the result $p=0$. It is obvious that this so-called proof is meaningless. 
Besides, Abel confused the concepts of the variable and the coefficients of equation. In Equation (41), $y$ is a variable. By solving the equation, $y$ can be expressed as the function of coefficients $d$ and $e$ which do not contain $y$. If we use Equation (42) to express $y$, the variable becomes $R^{1 / 3}$. In this case, $p$ and $p_{2}$ are the function of $d, e$ and cannot contain $R$. This is the basic principle of mathematics and cannot be violated. Otherwise the serious confusion and mistakes will be caused.

3) For the quartic equation

$$
y^{4}+b y^{3}+c y^{2}+d y+e=0
$$

Equation (33) becomes

$$
y=p+R^{1 / 4}+p_{2} R^{2 / 4}+p_{3} R^{3 / 4}
$$

On the other hand, let

$$
\begin{gathered}
\Delta_{1}=c^{2}-3 b d+12 e \\
\Delta_{2}=2 c^{3}-9 b c d+27 d^{2}+27 b^{2} e-72 c e \\
\Delta=\frac{2^{1 / 3} \Delta_{1}}{3\left[\Delta_{2}+\sqrt{-4 \Delta_{1}^{3}+\Delta_{2}^{2}}\right]^{1 / 3}}+\frac{\left[\Delta_{2}+\sqrt{-4 \Delta_{1}^{3}+\Delta_{2}^{2}}\right]^{1 / 3}}{3 \times 2^{1 / 3}}
\end{gathered}
$$

the four solutions of Equation (78) can be written as [11]

$$
\begin{aligned}
& y_{1}=-\frac{b}{4}-\frac{1}{2} \sqrt{\frac{b^{2}}{4}-\frac{2 c}{3}+\Delta}-\frac{1}{2} \sqrt{\frac{b^{2}}{4}-\frac{2 c}{3}-\Delta-\frac{-b^{3}+4 b c-8 d}{4 \sqrt{b^{2} / 4-2 c / 3+\Delta}}} \\
& y_{2}=-\frac{b}{4}-\frac{1}{2} \sqrt{\frac{b^{2}}{4}-\frac{2 c}{3}+\Delta}+\frac{1}{2} \sqrt{\frac{b^{2}}{4}-\frac{2 c}{3}-\Delta-\frac{-b^{3}+4 b c-8 d}{4 \sqrt{b^{2} / 4-2 c / 3+\Delta}}} \\
& y_{3}=-\frac{b}{4}+\frac{1}{2} \sqrt{\frac{b^{2}}{4}-\frac{2 c}{3}+\Delta}-\frac{1}{2} \sqrt{\frac{b^{2}}{4}-\frac{2 c}{3}-\Delta-\frac{-b^{3}+4 b c-8 d}{4 \sqrt{b^{2} / 4-2 c / 3+\Delta}}} \\
& y_{4}=-\frac{b}{4}+\frac{1}{2} \sqrt{\frac{b^{2}}{4}-\frac{2 c}{3}+\Delta}+\frac{1}{2} \sqrt{\frac{b^{2}}{4}-\frac{2 c}{3}-\Delta-\frac{b^{3}+4 b c-8 d}{4 \sqrt{b^{2} / 4-2 c / 3+\Delta}}}
\end{aligned}
$$

Let $p=-b / 4$ and $p_{1}=p_{2}=1 / 2$ as well as

$$
\begin{gathered}
R_{1}=\frac{b^{2}}{4}-\frac{2 c}{3}+\Delta \\
R_{2}=\frac{b^{2}}{4}-\frac{2 c}{3}-\Delta-\frac{-b^{3}+4 b c-8 d}{4 \sqrt{b^{2} / 4-2 c / 3+\Delta}}
\end{gathered}
$$

$R_{1}$ and $R_{2}$ contain the square root $\Delta$ of $b, c, d, e$. They are not the rational functions of $b, c, d, e$. More important one is that $R_{1}$ and $R_{2}$ are not symmetry function. Equation (82) can be simply written as

$$
\begin{aligned}
& y_{1}=p-p_{1} R_{1}^{1 / 2}-p_{2} R_{2}^{1 / 2} \\
& y_{2}=p-p_{1} R_{1}^{1 / 2}+p_{2} R_{2}^{1 / 2} \\
& y_{3}=p+p_{1} R_{1}^{1 / 2}-p_{2} R_{2}^{1 / 2} \\
& y_{4}=p+p_{1} R_{1}^{1 / 2}+p_{2} R_{2}^{1 / 2}
\end{aligned}
$$

On the other hand, take $m=4$, Equation (40) becomes 


$$
\begin{aligned}
& y_{1}=p+p_{1} R^{1 / 4}+p_{2} R^{2 / 4}+p_{3} R^{3 / 4} \\
& y_{2}=p+p_{1} \alpha R^{1 / 4}+p_{2} \alpha^{2} R^{2 / 4}+p_{3} \alpha^{3} R^{3 / 4} \\
& y_{3}=p+p_{1} \alpha^{2} R^{1 / 4}+p_{2} \alpha^{3} R^{2 / 4}+p_{3} \alpha R^{3 / 4} \\
& y_{4}=p+p_{1} \alpha^{3} R^{1 / 4}+p_{2} \alpha R^{2 / 4}+p_{3} \alpha^{2} R^{3 / 4}
\end{aligned}
$$

Equation (86) is completely different from Equation (85). For the quartic equation, Abel's solutions (33) and (40) do not hold. In fact, Abel's proof required that $m$ in Equation (32) was a prime number, so at least we can say that Abel's proof is not universal for it does not apply to the even degree equations with $m>2$.

4) Because Equation (33) cannot hold for the cubic and quartic equations, we have no reasons to insist that Equations (33) and (40) are effective for the quintic equation. It can be imagined that the solution of quintic equation would be much more complex than the quartic equation. It can see that the basic forms of quatratic, cubic and quartic equations are greatly different. The form of quintic equation is difficult to forecast, unless we solve the equation and obtain it really.

V) Equations (36) and (37) also have the problems. Equation (36) is only a definition, or an identical equation, in which both $\mathrm{z}$ and $\mathrm{R}$ are unknown. As we known, as a meaningful equation which can be solved, it should contain known quantity and unknown quantity. We use known quantity to represent unknown quantity. But (36) is not such equation.

Abel thought that Equations (36) and (37) had the common root. This is wrong. In fact, Equation (37) is only the variable substitution of Equation (34) by using Equation (36). By means of the solution of Equation (37), we can determine $\mathrm{z}$ and then also determine R. Equation (37) can be solved independently and Equation (36) is unnecessary. It does not make sense to combine them for solution. Equations (38) and (39) do not exist.

In brief, because Equations (33) and (34) are untenable in general, the first part of Abel's proof in the paper in 1824 was wrong. The second part became meaningless. We do not need to discuss it any more.

\section{Brief Introduce to the Galois's Theory}

\subsection{The Symmetry between the Coefficients and the Roots of Algebraic Equations}

General $n$ degree algebraic equation can be written as

$$
f(x)=x^{n}+a_{n-1} x^{n-1}+a_{n-2} x^{n-2}+\cdots+a_{1} x+a_{0}=0
$$

Here the coefficients $a_{i} \in Q$ are rational numbers in general. According to the Gauss basic theorem, there are $\mathrm{n}$ roots $x_{1}, x_{2}, \cdots, x_{n}$. So Equation (87) can be written as

$$
\left(x-x_{n}\right)\left(x-x_{n-1}\right)\left(x-x_{n-2}\right) \cdots\left(x-x_{1}\right)=0
$$

By expanding the multiplication of Equation (88) and comparing it with Equation (87), the relation between the roots and the coefficients are [12] 


$$
\begin{gathered}
x_{1}+x_{2}+x_{3}+\cdots+x_{n}=-a_{n-1} \\
x_{1} x_{2}+x_{1} x_{3}+\cdots+x_{1} x_{n}+x_{2} x_{3}+x_{2} x_{4}+\cdots=a_{n-1} \\
\vdots \\
x_{1} x_{2} x_{3} \cdots x_{n}=(-1)^{n} a_{0}
\end{gathered}
$$

Equation (89) is so-called as the Vieta's formulas, of which right side is constants. Exchanging $x_{1}, x_{2}, \cdots, x_{n}$ in the left sides of Equation (89), for example, let $x_{1} \Leftrightarrow x_{2}$ or $x_{i} \Leftrightarrow x_{k}$, its right side are unchanged. So there are symmetries between the roots and coefficients. In the words of permutation group, there exists $S_{n}$ symmetry for the algebraic equations.

\subsection{The Galois's Theory of Solvable Group $G_{n}$}

By introducing the concept of radical domain extension, the symmetry of permutation group $S_{n}$ was transformed into the tower structure of Galois's group $G_{n}$ of radical expansion domain. Basic on it, the radical solutions of equations were discussed.

According to the Galois theorem, the necessary and sufficient condition that an algebraic equation has the radical solution is that that the corresponding Galois group is the solvable group. The definition of the solvable group is below [12]. Suppose that $S_{n}$ is the finite permutation group, there exist a series of normal subgroup which form the tower structure

$$
S_{n} \sim G_{n} \geq G_{1} \geq G_{2} \geq \cdots \geq G_{r}=I
$$

where $I$ is the unit element, $G_{i+1}$ is the normal subgroup of $G_{i}$. If each quotient group $G_{i} / G_{i+1}$ in the series is exchangeable, Equation (90) is called as the series of solvable group and $G_{n}$ is called as solvable group. According to Galois theory, the tower structure of normal subgroup shown in Equation (90) was equivalent to decompose Equation (87) into the multiplying forms of each single item of Equation (88).

Because the permutation group $S_{5}$ corresponding quintic equation was a single group without true normal subgroup, the tower structure Equation (90) did not exist, Galois thought the quintic equation had no radical solution. Because $S_{n}$ group always has a subgroup $S_{5}$ when $n>5$, the higher degree equations were also considered to have no radical solutions.

\subsection{The Galois's Theory of Radical Extension}

In order to apply the solvable group theory to solve the algebraic equations, we need to introduce the concept of radical expansion [12]. It should be pointed out that Galois didn't really use the concept of radical expansion in his original paper, but there was such an idea. The strict radical extension theory and its most practical applications were the result of the improvement of Galois's early theory by later mathematicians. As a concept of number domain, the concept of expansion domain needs to meet some rules, but we do not go into details here.

What was discussed in the Galois's theory was the irreducible polynomial on $Q\left(a_{i}\right)$ in general, in which the equation's coefficients $a_{i} \in Q$ were rational 
numbers. The processes to find the roots of equations were considered as the expansion process of radical domain, or the process to add the roots in $Q\left(a_{i}\right)$. For example, suppose that we have obtained an irrational root $x_{1} \sim \sqrt{b}$ by solving the equation. Adding it in $Q\left(a_{i}\right)$, we get $Q\left(a_{i}, \sqrt{b}\right)$. We call $Q\left(a_{i}, \sqrt{b}\right)$ as the extension of radical domain.

By solving the equation further, we obtain more roots, for example, we get $x_{1} \sim \sqrt{g+\sqrt{b}}$. The radical domain $Q\left(a_{i}, \sqrt{b}\right)$ was extended into

$Q\left(a_{i}, \sqrt{b}, \sqrt{g+\sqrt{b}}\right)$, and so do. At last, all roots are founded, the whole root domain is described by $E=E\left(x_{1}, x_{2}, \cdots, x_{n}\right)$.

According to the theory of Galois, if the radical extension can be carried out for an equation, the equation is considered to have radical solution. If the radical extension cannot be carried out, the equation is considered to have no radical solution. The quintic equation is considered to have no radical solutions because its radical expansion is considered impossible.

\subsection{The Automorphism Mapping Group GalE/Q}

After the concept of radical extension is introduced, in order to establish the relation between the radical extension and the permutation group, the automorphism mapping concept of radical extension is needed. Based on it, the automorphism mapping group GalE/Q is established. Then, through the Galois's correspondence theorem [13], GalE/ $Q$ was connected with the solvable group $G_{n}$, or using $G_{n}$ to replace $G a l E / Q$. By considering whether or not $G_{n}$ had the tower structure, Galois judged whether or not the equation had radical solution. So the automorphism mapping is the core concept of Galois's theory. It is the key to understanding the theory of Galois. We should make clear the logic relation here.

The operator $\sigma$ is used to represent the automorphism mapping. It changes the root to another root of the equation or keeps the root self unchanged. Meanwhile, it does not change the coefficients of equation [12]. Suppose that $x_{i}$ is a root of equation (87), $E$ is the root domain, we have $f\left(x_{i}\right)=0$ and define

$$
\begin{gathered}
\sigma\left(x_{i}\right)=x_{j} \mid x_{i}, x_{j} \in E \\
\sigma f\left(x_{i}\right)=\sum_{k=1}^{n} a_{k} \sigma\left(x_{i}^{k}\right)=\sum_{k=1}^{n} a_{k}\left(\sigma x_{i}\right)^{k}=\sum_{k=1}^{n} a_{k} x_{j}^{k}=0
\end{gathered}
$$

If the roots are known, by means of Equation (92), the concrete form of operator $\sigma$ can be obtained. It is proved that the automorphism mapping forms group, called as the Galois group GalE/Q of radical extension.

By the complex reasoning process, it was proved that the automorphism mapping group GalE/ $Q$ was equivalent to the permutation group $S_{n}$ [12]. More complicated reasoning indicated that the automorphism mapping group was equivalent to the subgroup $G_{n}$ of permutation group $S_{n}$ (For example, the normal subgroup $A_{3}$ of $S_{3}$ ) [13]. We cannot cite them any more here, but can take some practical example. For the simplest quadratic equation 


$$
f(x)=x^{2}+a x+b=0
$$

Its two roots are

$$
x_{1}=\frac{1}{2}\left(-a+\sqrt{a^{2}-4 b}\right), x_{2}=\frac{1}{2}\left(-a-\sqrt{a^{2}-4 b}\right)
$$

The process of radical extension is $Q(a, b) \rightarrow Q\left(a, b, \sqrt{a^{2}-4 b}\right)$. Let $I$ be identical transformation, the group element (12) represent the permutation $x_{1} \leftrightarrow x_{2}$, we have $\sigma \sim S_{2}=\{I,(12)\}$. It is obvious that the Galois group GalE/Q is equivalent to $S_{2}$. There are four roots for the quartic equation

$$
f(x)=\left(x^{2}-a\right)\left(x^{2}-b\right)=0
$$

There are $x_{1}=\sqrt{a}, x_{2}=-\sqrt{a}, x_{3}=\sqrt{b}$ and $x_{4}=-\sqrt{b}$. Let group element (12) represent $x_{1} \leftrightarrow x_{2}$, the group element (34) represent $x_{3} \leftrightarrow x_{4}$, we have $\sigma \sim\{I,(12),(34),(12)(34)\}$. It is just the Klein subgroup $V_{4}$ of $S_{4}$ [12].

The concrete form of GalE/ $Q$ group is difficult to be calculated for high degree equation, because we should know the solution of the equation. However, it is hard work to obtain the solutions of high degree equations. So the concrete forms of GalE/ $Q$ of high degree equations actually are unknown.

To solve this problem, the so-called Galois corresponding relation was proposed [13]. It assumed that the Galois solvable group $G_{n}$ can be used to replace the radical extension group $G a l E / Q$. Whether the $G_{n}$ group has the tower structure is equivalent to whether radical extension can be carried out.

Because the $S_{5}$ group has no real normal subgroup, $G_{5}$ is not the solvable group without the tower structure, the radical extension processes of quintic equations are considered impossible, so the quintic equations are considered to have no radical solutions.

\section{The Problems Existing in Galois's Theory}

\subsection{The General Description of the Problem of Galois's Theory}

It is pointed out that Galois only assumed that the quartic equation had no radical solution without really proving it. According to the definition, the automorphism mapping operator should be acted on the roots of equations. However, in order to prove that the theory was effective for the general cubic and quartic equations, some relations between the roots of equation had to be used to replace the roots themselves. This is violated the definition of automorphism mapping concept and led to the ineffectiveness of the proof.

The theory of radical extension is inconsistent with practical process of solving equation. The tower structure of Galois's solvable group does not exist in the practical processes of solving equations. It is only something formal without practicability. It was also unsuccessful using the theory of solvable group to describe the resolvents of general cubic and quartic equations.

The Galois's theory is a paradox. The theory admits the existence of permutation symmetry of $S_{5}$ group. This fact indicates that it admits the exis- 
tences of the Vieta formula and the solutions of quartic equations. It is illogical to argue that the radical solutions are impossible simply because the group has no true normal subgroup, unless the Vieta's formula and $S_{5}$ group do not exist too. The absence of true normal subgroup only indicates that there is no symmetry of normal subgroup between the roots. It does not mean the absence of radical solutions.

In fact, Galois only proved that the alternating group $A_{n}(n>5)$ was a single groups, but had not proved that the permutation group $S_{n}(n>5)$ was also a single groups. For example, $S_{5}$ is a true normal subgroup of $S_{9}$, but it is not the only true normal subgroup. It is only an exception without true normal subgroup. All permutation groups $S_{n}$ which contain the non-prime cyclic groups as subgroups must not be single groups. How can we say that there are no radical solutions for all equations with $n>5$ degrees?

\subsection{Galois' Theory Cannot Explain Tan Jianer's Solution of Quintic Equation}

It is obvious that Galois' theory cannot explain Tang Jianer's solution of quintic equation. Take Equation (11) as a example, the five solutions described by Equations (10) and (19) still satisfied the Vieta's Formula (89). There is the symmetry of $S_{5}$ group between the roots and coefficients. Thought there is a relation $p^{2}=5 r$ for coefficients, it does not change the symmetry of $S_{5}$ group. Because $S_{5}$ has no true subgroup and the tower structure of soluble group, according to Galois's theory, Equation (11) has no radical solutions. However, Tang Jianer solved the quintic equation and obtained the radical solutions.

\subsection{The Solutions of High Degree Equations with Number Coefficients}

What the Galois's theory discussed was the equations irreducible on $Q$ with arbitrary coefficients. However, there are great numbers of equations with number coefficients in practice. It is very difficult to judgment whether these equations are reducible or irreducible. At present, they are actually classified to the equations unsolvable. But it is not true, for example, for the quintic equation below

$$
\begin{aligned}
& a_{5} x^{5}+a_{4} x^{4}+a_{3} x^{3}+a_{2} x^{2}+a_{1} x+a_{0} \\
& =3 x^{5}+7 x^{4}-18 x^{3}-49 x^{2}+17 x+14=0
\end{aligned}
$$

Based on the relation between the roots and the coefficients, Zheng Liangfei obtained a solution $x_{1}=2 / 3$ by using his special method, and reduced the equation into the quartic equation. Then by solving the quartic equation, he obtained the solutions of the quintic equation [8]. The results are

$$
x_{1}=\frac{2}{3}, x_{2,3}=1 \pm \sqrt{2}, x_{4,5}=\frac{-5 \pm i \sqrt{3}}{2}
$$

Among them, three are real numbers and two are complex numbers. In three real numbers, two are irrational numbers and one is rational number. Because there is a rational number's solution, Equation (96) is reducible on $Q$. However, 
if the equation has not be solved, we cannot judge whether it is reducible on $Q$. In fact, in many situations, the processes to judge whether or not an equation is reducible on $Q$ is actually the processes to solve the equation. Therefore, it is actually meaningless to assume a high degree equation to be reducible on $Q$.

As we known that there is a theorem called the Eisenstein criterion which can be used to judge the reducibility of an equation. But it is only a criterion of sufficient condition, not a criterion necessary condition. Its application is limited. For example, for Equation (96), we have $a_{0}=14, a_{1}=17, a_{2}=-49, a_{3}=-18$, $a_{4}=7, a_{5}=3$. According to the Eisenstein criterion, if we can find a prime $p$ it is divisible into $a_{0}, a_{1}, a_{2}, a_{3}, a_{4}$, but cannot be divisible into $a_{5}$. Meanwhile, $p^{2}$ cannot be divisible into $a_{0}$, Equation (96) is reducible on $Q$. It is obvious that we cannot find such a prime, so we cannot judge whether or not Equation (96) is reducible on $Q$. however, practical result is that Equation (96) has a solution of real number $x_{1}=2 / 3$.

At present, we have no generally effective method to judge the reducibility of general algebraic equation. According to common understanding, Equation (96) may be considered impossible to solve.

Zheng Liangfei did a lot of work on the solutions of quartic equations with number coefficients and published a book titled "The solutions of Quantic Equations" [8]. Same effective methods were proposed to deal with various quantic equations with number coefficients. According to the opinion of Zheng Liangfei, any high degree equations with number coefficients can be solved in principle.

\subsection{The Radical Solutions of High Degree Cyclotomic Equation}

The equation with the form $x^{n}-1=0$ is called as the cyclotomic equation which can be resolved as

$$
x^{n}-1=(x-1)\left(x^{n-1}+x^{n-2}+\cdots+1\right)=0
$$

So it is a reducible polynomial on $Q$ with a rational number solution $x=1$. The roots of Equation (98) are called as the source roots with the form

$$
x_{k}=\omega^{k}=\mathrm{e}^{i 2 \pi k / n}=\cos 2 \pi k / n+i \sin 2 \pi k / n, k=0,1,2, \cdots, n-1
$$

Taking $n=6$, Equation (98) can be written as two equations. One is $x-1=0$ and another is a quartic equation

$$
x^{5}+x^{4}+x^{3}+x^{2}+x+1=0
$$

The solution of Equation (100) is

$$
x_{k}=\omega^{k}=\cos (2 \pi k / 6)+i \sin (2 \pi k / 6), \quad k=1,2,3,4,5
$$

So $x_{k}$ are not rational numbers, for example, $x_{1}=1 / 2+i \sqrt{3} / 2$. It indicates that the radical solutions of (100) exist. However, the Vieta's formula of Equation (100) satisfies $S_{5}$ group which is a commutative cyclic group. According to the Galois's group theory, the cyclic group is not a solvable group without true subgroup [12]. But Equation (100) has radical solutions. Obviously, the Galois's theory is invalid for the quartic equation (100). 


\subsection{The Theory of Radical Extension of General Cubic Equation Is Invalid}

The theory of radical extension is only a formal thing. In the actual processes to solve equations, the theory of radical tower extension is not obeyed. Although there is a large amount of literature to discuss the constructions of quadratic equations and some reducible cubic and quadric equations, only there is a little literature to discuss the radical tower constructions of general and irreducible cubic and quadric equations.

The following is an example to construct the radical tower of general cubic equation cited from reference [12]. It can be seen that this proof is far-fetched and illogical. The general cubic equation is written as

$$
f(x)=x^{3}+p x+q=0
$$

where $p, q \in Q$ are rational numbers. The three solutions of Equation (102) are

$$
\begin{aligned}
& x_{1}=\sqrt[3]{-q / 2+\sqrt{(q / 2)^{2}+(p / 3)^{3}}}+\sqrt[3]{-q / 2-\sqrt{(q / 2)^{2}+(p / 3)^{3}}} \\
& x_{2}=\omega \sqrt[3]{-q / 2+\sqrt{(q / 2)^{2}+(p / 3)^{3}}}+\omega^{2} \sqrt[3]{-q / 2-\sqrt{(q / 2)^{2}+(p / 3)^{3}}} \\
& x_{3}=\omega^{2} \sqrt[3]{-q / 2+\sqrt{(q / 2)^{2}+(p / 3)^{3}}}+\omega \sqrt[3]{-q / 2-\sqrt{(q / 2)^{2}+(p / 3)^{3}}}
\end{aligned}
$$

Here

$$
\begin{aligned}
& \omega=\mathrm{e}^{i 2 \pi / 3}=\cos 2 \pi / 3+i \sin 2 \pi / 3=-1 / 2+i \sqrt{3} / 2 \\
& \omega^{2}=\mathrm{e}^{i 4 \pi / 3}=(-1 / 2+i \sqrt{3} / 2)^{2}=-1 / 2-i \sqrt{3} / 2
\end{aligned}
$$

The Vita's formula of Equation (102) corresponds to the permutation group $S_{3}$. Its six elements are

$$
S_{3}=\{I,(12),(13),(23),(123),(132)\}
$$

The unique true subgroup of $S_{3}$ is $A_{3}$. It is a normal subgroup with three elements

$$
A_{3}=\{I,(123),(132)\}
$$

The group elements (123) and (132) are even permutations. According to the Galois's theory, the tower structure of solvable group is

$$
S_{3} \sim G_{3} \geq A_{3} \geq I
$$

It is seen from the relation (109) that there are two steps in the process $G_{3} \rightarrow I$ according to the solvable group theory. However, according the theory of radical extension, there are three additions of roots. The first extension is to add the first level root symbol with

$$
Q(p, q) \rightarrow Q_{1}\left(p, q, \sqrt{(q / 2)^{2}+(p / 3)^{3}}\right)
$$

The second extension is to add the second level root symbol with

$$
Q_{1} \rightarrow Q_{2}\left(p, q, \sqrt[3]{-q / 2 \pm \sqrt{(q / 2)^{2}+(p / 3)^{3}}}\right)
$$


The third extension is to add the imaginary number and root symbol $\omega \sim i \sqrt{3}$

$$
Q_{2} \rightarrow Q_{3}\left(p, q, i \sqrt{3}, \sqrt[3]{-q / 2 \pm \sqrt{(q / 2)^{2}+(p / 3)^{3}}}\right)
$$

Therefore, the tower structure of radical extension should be $Q \leq Q_{1} \leq Q_{2} \leq Q_{3}=E$, different from that shown in the Formula (109).

In order to make the practical process of radical extension satisfying the Galois's theory, a very complicated and full of holes proof was proposed in reference [12]

1) At first, $\omega \sim i \sqrt{3}$ is added in the basic field $F$, to let

$$
F=F(r, p, q, i \sqrt{3})
$$

Meanwhile, the radical field is

$$
E=E\left(x_{1}, x_{2}, x_{3}\right)=F^{\prime \prime}\left(r, p, q, i \sqrt{3}, x_{1}, x_{2}, x_{3}\right)
$$

2) Suppose that the Galois's group was $G(E / F)=S_{3}$. The tower structure of solvable group was $G=S_{3} \geq A_{3} \geq I$. The tower structure of radical extension is $F(r, p, q, i \sqrt{3}) \leq B \leq E\left(x_{1}, x_{2}, x_{3}\right)$, where $B=\operatorname{Inv} A_{3}$ (Inv represents invariable) and $A_{3}=G(E / B)$ was three-degree cyclic group. Suppose that $B$ was the normal extension of $F^{\prime}, E$ was regarded as the three-time cyclic extension of $B$ with the degree $\left|A_{3}\right|=3$.

3) Introducing the criteria formula of cubic equation

$$
\Delta=\left(x_{1}-x_{2}\right)\left(x_{2}-x_{3}\right)\left(x_{3}-x_{1}\right)
$$

Because $\Delta$ is unchanged under the even permutation $A_{3}=\{I,(123),(132)\}$, so we have $\Delta \in B$. Because $S_{3}$ cannot keep unchanged under the odd permutation, so we have $\Delta \notin F$. Let $D=\Delta^{2}$, due to that $D$ is unchanged under odd permutation, so we have $D \notin F^{\prime}$. It can be proved that

$$
D=\Delta^{2}=-27 q^{2}-4 p^{2}=a
$$

4) because $\Delta \notin F$ and $\Delta \in B$, the extension of $F$ is $F^{\prime}(\Delta)$. It means $F \leq F^{\prime}(\Delta) \leq B$. However, because $D=\Delta^{2}=a \in F$, where $\Delta$ is the root of quation $x^{2}-a=0$, so we have $\left[F^{\prime}(\Delta): F\right]=2$. Because $G(B / F)=G(E / F) / G(E / B) \approx S_{3} / A_{3}$, we get $[B: F]=|G(B / F)|=\left|S_{3}\right| /\left|A_{3}\right|=2$. Therefore, it is proved with $B=F^{\prime}(\Delta)$.

5) If all three roots $x_{1}, x_{2}, x_{3}$ belong to $B$, we have $B=E$. But this is contradictory. So we let $x_{1} \notin B$ and $B^{\prime}=B\left(x_{1}\right) \geq B$. Because $\left|A_{3}\right|=3$, it indicates that we cannot inset other subgroup in $A_{3} \geq I$, which means $B\left(x_{1}\right)=E$. Thus, the tower structure of radical field is $F \leq B \leq E$. Writing it more explicitly, the result is

$$
F(r, p, q, i \sqrt{3}) \rightarrow B\left(r, p, q, i \sqrt{3}, x_{2}, x_{3}\right) \rightarrow E\left(r, p, q, i \sqrt{3}, x_{1}, x_{2}, x_{3}\right)
$$

Obviously, this extension process has serious problem. Firstly, it is completely irrational to add $\omega \sim i \sqrt{3}$ in the base field $F$. The precondition of Galois's 
theory is that the base field is rational number field without containing radical symbol and imaginary number. The reason why the Formula (113) is used to describer the base field is that the solutions of cubic equation contain multiple roots and imaginary numbers, but $S_{3}$ group can only accommodate a middle field so that it cannot be extended according to the Galois's model. Thus, $i \sqrt{3}$ had to be added in the base field to justify the theory

Secondly, this method has not expressed how to obtain $A_{3}$ group through the automorphism mapping. The middle field $B$ contains $x_{2}, x_{3}$, but not contain $x_{1}$. The automorphism mapping means that automorphism group only contains the group element $(2,3)$ between the exchange of roots $x_{2}$ and $x_{3}$, do not contain group elements $(1,2,3)$ and $(1,3,2)$ between the exchange of roots $x_{1}$ and $x_{2}, x_{3}$. In fact, because $A_{3}$ group contain index $1,2,3$, the first extension cannot exclude any one among the solution $x_{1}, x_{2}, x_{3}$. Therefore, the radical tower extension field is impossible.

Besides, it is impossible to explain why we need to introduce the relation (116). The second degree equation $x^{2}-a=0$ has nothing to do with radical extension process of the cubic equation. Using it to construct meddle field is illegal. In the practical process of solving equation, we find one solution $x_{1}$, then obtain another two. This is different from what described above. The Galois's theory of radical extension is invalid for the general cubic equation.

\subsection{The Symmetry Change of Permutation Group in the Practical Radical Extension}

According to Equations (103)-(105), the first solution of cubic equation is $x_{1}$. The first radical extension is from rational number to irrational number. We have

$$
Q(p, q) \rightarrow Q_{1}\left(p, q, \sqrt[3]{-q / 2 \pm \sqrt{(q / 2)^{2}+(p / 3)^{3}}}\right)
$$

Then we obtain the second solution $x_{2}$ and the third solution $x_{3}$. The second radical extension is from real number field to imaginary number field

$$
Q_{1} \rightarrow Q_{2}\left(p, q, i \sqrt{3}, \sqrt[3]{-q / 2 \pm \sqrt{(q / 2)^{2}+(p / 3)^{3}}}\right)
$$

Thought two extensions, the right side of Equation (119) contains all roots of the equation. The tower structure of extension field is $Q \leq Q_{1} \leq Q_{2}=E$. It indicates that the practical process of solving cubic equation is not through the additions of radical symbols one by one. The double root is obtained at one time, then entering the imaginary number field by adding $i \sqrt{3}$.

More generally, suppose that we have obtained a root $x_{1}$ by solving the equation, Equation (102) can be written as

$$
\left(x-x_{1}\right)\left(x^{2}+a x+b\right)=0
$$

By developing Equation (120), we obtain

$$
x^{3}+\left(a-x_{1}\right) x^{2}+\left(b-a x_{1}\right) x-b x_{1}=0
$$


Comparing Equation (121) with Equation (102), we get $a-x_{1}=0, b-a x_{1}=p$ and $b x_{1}=-q$. So we get $a=x_{1}$ and $b=-q / x_{1}=p+x_{1}^{2}$. In order to obtain another solution, according to (120), we have

$$
\left(x^{2}+a x+b\right)=0
$$

Equation (122) is the second degree equation. Its Vieta'S formula satisfies the symmetry $S_{2}=\{I,(12)\}$. It is obvious that $S_{2}$ is not $A_{3}$ group. So in the practical process of solving equation, subgroup $A_{3}$ does not work. The change of symmetry between the coefficients and the roots of equation is $S_{3} \rightarrow S_{2} \rightarrow I$, rather then $S_{3} \geq A_{3} \geq I$. The solvable group theory of Galois does not match the practical process of solving equation.

\subsection{It Is Illegal to Use the Relations between Roots Replacing the Roots to Construct Automorphism Mapping Operator}

In common textbooks, a fatal mistake is made to prove the effectiveness of Galois's theory. In order to construct the radical extension groups, the automorphism mapping operators are acted on the relations between the roots of equations, rather than acted on the roots themselves. The method violates the definition of Equation (92) and is invalid. Unfortunately, this problem has always been ignored.

To illustrate the problem, the quartic equation is taken as an example. This example also shows that Galois's theory of radical extension is invalid for the quartic equation. Take a simplified quartic equation [14]

$$
f(x)=x^{4}+p x^{2}+q=0
$$

where $p, q \in Q$. Let $x^{2}=y$, Equation (123) becomes the second degree equation $y^{2}+p y+q=0$. Its two solutions are

$$
y_{1}=\frac{-p+\sqrt{p^{2}-4 q}}{2}, y_{2}=\frac{-p-\sqrt{p^{2}-4 q}}{2}
$$

Therefore, by taking square roots, the four solutions of Equation (123) are

$$
\begin{aligned}
& x_{1}=\sqrt{\frac{-p+\sqrt{p^{2}-4 q}}{2}}, \quad x_{2}=-\sqrt{\frac{-p+\sqrt{p^{2}-4 q}}{2}} \\
& x_{3}=\sqrt{\frac{-p-\sqrt{p^{2}-4 q}}{2}}, \quad x_{4}=-\sqrt{\frac{-p-\sqrt{p^{2}-4 q}}{2}}
\end{aligned}
$$

In order to prove that this process satisfied the Galois's theory, the relations $x_{1}+x_{2}=0$ and $x_{3}+x_{4}=0$ are considered. They are unchanged under following 8 replacements, called as the early Galois group [14]

$$
B=\{I,(12),(34),(12)(34),(13)(24),(14)(23),(1423),(1324)\}
$$

On the other hand, based on Equation (125), we have

$$
\begin{aligned}
& x_{1}^{2}-x_{3}^{2}=\sqrt{p^{2}-4 q} \\
& x_{2}^{2}-x_{4}^{2}=\sqrt{p^{2}-4 q}
\end{aligned}
$$

The right sides of Equation (127) and (128) are completely the same, so we 
only need to consider one of them. It can be verified that Equation (127) can keep unchanged under the first four permutations $I,(12),(34),(12)(34)$ of $B$ shown in Equation (126).

By adding the relation of Equation (127) in the base field $Q(p, q)$, the radical extension is obtained with

$$
Q(p, q) \rightarrow Q^{\prime}\left(p, q, \sqrt{p^{2}-4 q}\right)
$$

However, Equation (127) cannot keep unchanged under the last four permutations (13) (24), (14) (23), (1423) and (1324). In order to prove the effectiveness of radical extension, another relation is introduced with

$$
x_{3}-x_{4}=2 \sqrt{\frac{-p-\sqrt{p^{2}-4 q}}{2}}
$$

Equation (130) is invariable to the group elements $I,(12)$ which can be considered as a subgroup of Equation (126). The root in the right side of Equation (130) is added in the field $Q^{\prime}\left(p, q, \sqrt{p^{2}-4 q}\right)$, the second radical extension is obtained with

$$
Q^{\prime}\left(p, q, \sqrt{p^{2}-4 q}\right) \rightarrow Q^{\prime \prime}\left(p, q, \sqrt{p^{2}-4 q}, \sqrt{\left(-p-\sqrt{p^{2}-4 q}\right) / 2}\right)
$$

Then, introducing another relation

$$
x_{1}-x_{2}=2 \sqrt{\frac{-p+\sqrt{p^{2}-4 q}}{2}}
$$

It is invariable to the permutation elements $I,(34)$, so the radical field of Equation (131) is enlarged further with

$$
\begin{aligned}
& Q^{\prime \prime}\left(p, q, \sqrt{p^{2}-4 q}, \sqrt{\left(-p-\sqrt{p^{2}-4 q}\right) / 2}\right) \\
& \rightarrow Q^{\prime \prime \prime}\left(p, q, \sqrt{p^{2}-4 q}, \sqrt{\left(-p-\sqrt{p^{2}-4 q}\right) / 2}, \sqrt{\left(-p+\sqrt{p^{2}-4 q}\right) / 2}\right)=E
\end{aligned}
$$

$E$ is just the radical field of Equation (123), so the Galois's theory is regarded to be effective for the quartic equation.

However, there are many problems in the discussions above.

1) At first, the Equation (123) is only a special quartic equation, essentially a second degree equation. The solution (83) of general quartic equation (78) is very complex. It is impossible to use the Galois's theory of radical extension above to described it.

2) The Vieta's formula of quartic equation satisfies the symmetry of $S_{4}$ group with 24 elements

$$
\begin{aligned}
S_{4}= & \{I,(12),(13),(14),(23),(24),(34),(12)(34),(13)(24), \\
& (14)(23),(123),(132),(124),(142),(134),(143),(324), \\
& (243),(1234),(1243),(1324),(1342),(1423),(1432)\}
\end{aligned}
$$

According to the definition of normal subgroup, the tower structure of Galois solvable group is 


$$
S_{4} \sim G_{4} \geq A_{4} \geq V \geq I
$$

Here $A_{4}$ is the biggest normal subgroup of $S_{4}$ with 12 elements

$$
\begin{aligned}
A_{4}=\{ & I,(12)(34),(13)(24),(14)(23),(123),(132), \\
& (124),(142),(134),(143),(324),(243)\}
\end{aligned}
$$

$V$ is the Klein group with 4 elements

$$
V=\{I,(12)(34),(13)(24),(14)(23)\}
$$

Obviously, $B$ described by Equation (126) does not belong to any subgroup of $S_{4}$ shown in Equation (134), saying nothing of a normal subgroup. So it is invalid using the symmetry shown in Equation (126) to prove the effectiveness of Galois's theory.

3) The relation $x_{1}+x_{2}=0$ and $x_{3}+x_{4}=0$ has nothing to do with the process of solving equation. According to the radical extension theory of Galois, the automorphism mapping operator acts on a root of an equation and change it into another root of the same equation, or makes this root unchanged. However, $x_{1}+x_{2}$ and $x_{3}+x_{4}$ are not the roots of original equation. Substituting them in Equation (123), we get $\left(x_{1}+x_{2}\right)^{4}+p\left(x_{1}+x_{2}\right)^{2}+q=q \neq 0$. So it is illegal using $x_{1}+x_{2}=0$ and $x_{3}+x_{4}=0$ to construct the Galois's group shown in Equation (126).

Similarly, $x_{1}-x_{2}$ and $x_{3}-x_{4}$ are not the roots of Equation (123). It is illegal to act the automorphism mapping operator on them. It is meaningless by adding the right side of equations (130) and (132) to prove the theory of radical extension of automorphism mapping, also having nothing to do with the practical process of solving equations.

4) It is actually a common method to use the algebraic relations of equation's roots to replace the roots in order to prove the effectiveness of Galois's theory at present. It not only confuses the concepts and but also violates the uniqueness of theory. For example, by using the roots $x_{1}, x_{2}, x_{3}, x_{4}$, we can construct $x_{1} x_{2}$, $x_{1}^{2} x_{2}$ and $x_{1}^{2} x_{3}+x_{4}$ and so on. We have no reason to use someone, do not use another one.

5) The tower structure of Galois group cannot be formed by means of this method. The radical extensions of Equations (127) and (128) satisfy following permutations group

$$
B_{1}=\{I,(12),(34),(12)(34)\}
$$

The radical extensions of Equations (130) and (132) satisfy following permutations group

$$
B_{2}=\{I,(12)\}, B_{3}=\{I,(34)\}
$$

The relations between $B$ and $B_{2}$ or $B_{3}$ are not that between the groups and its normal subgroups.

Because the radical extension theories of general cubic and quartic equations cannot match with the practical process, we have no reason to think that the 
solvable group theory of Galois is effective for the quintic equation. For the high degree equations, the possible symmetry train of permutation group should be

$$
S_{n} \rightarrow S_{n-1} \rightarrow \cdots \rightarrow S_{2} \rightarrow I
$$

That is to say, the relation of roots and coefficients of an equation only has the symmetry of $S_{n}$. The tower structure of Galois solvable group $G_{n}$ corresponding to the subgroup chain of $S_{n}$ group does not exist in the practical processes of solving equations.

\subsection{The Resolvent of Cubic Equation}

Lagrange introduced the concepts of permutation group and resolvent to solve the cubic and quadric equation in 1770 [12]. The effectiveness of Galois's theory of solvable group also needs to be verified by using it to construct the resolvent of lower degree equations.

The quadratic equation is most simple, its symmetry group $S_{2}$ has only two elements $I$ and (12). We do not need to discuss it any more. For the resolvent of cubic equation (102), following two functions are used

$$
\begin{aligned}
& \alpha=x_{1}+\omega x_{2}+\omega^{2} x_{3} \\
& \beta=x_{1}+\omega x_{3}+\omega^{2} x_{2}
\end{aligned}
$$

Here $x_{1}, x_{2}, x_{3}$ are three roots of cubic equation described by Equations (103)-(105). It is proved that the second degree equation with the roots $\alpha^{3}$ and $\beta^{3}$ is the Lagrange resolvent of cubic equation. We have [15]

$$
\begin{aligned}
\alpha^{3}+\beta^{3}= & \left(x_{1}+\omega x_{2}+\omega^{2} x_{3}\right)^{3}+\left(x_{1}+\omega x_{3}+\omega^{2} x_{2}\right)^{3} \\
= & 2\left(x_{1}^{3}+x_{2}^{3}+x_{2}^{3}\right)+3\left(\omega+\omega^{2}\right)\left(x_{1}^{2} x_{2}+x_{2}^{2} x_{3}+x_{3}^{2} x_{1}+x_{1}^{2} x_{3}\right)^{3} \\
& +12 x_{1} x_{2} x_{3}
\end{aligned}
$$

Due to $1+\omega+\omega^{2}=0$, so $\omega+\omega^{2}=-1$, based on Equation (143), we have

$$
\begin{aligned}
\alpha^{3}+\beta^{3}= & 2\left[\left(x_{1}+x_{2}+x_{3}\right)^{3}-3\left(x_{1}^{2} x_{2}+x_{2}^{2} x_{3}+x_{3}^{2} x_{1}+x_{1}^{2} x_{3}\right)-6 x_{1} x_{2} x_{3}\right] \\
& -3\left(x_{1}^{2} x_{2}+x_{2}^{2} x_{3}+x_{3}^{2} x_{1}+x_{1}^{2} x_{3}\right)+12 x_{1} x_{2} x_{3} \\
= & 2\left(x_{1}+x_{2}+x_{3}\right)^{3}-9\left(x_{1}^{2} x_{2}+x_{2}^{2} x_{3}+x_{3}^{2} x_{1}+x_{1}^{2} x_{3}\right) \\
= & 2\left(x_{1}+x_{2}+x_{3}\right)^{3}-9\left[\left(x_{1} x_{2}+x_{1} x_{3}+x_{2} x_{3}\right)\left(x_{1}+x_{2}+x_{3}\right)-3 x_{1} x_{2} x_{3}\right] \\
\alpha \beta= & \left(x_{1}+\omega x_{2}+\omega^{2} x_{3}\right)\left(x_{1}+\omega x_{3}+\omega^{2} x_{2}\right) \\
= & \left(x_{1}^{2}+x_{2}^{2}+x_{2}^{2}\right)+\left(\omega+\omega^{2}\right)\left(x_{1} x_{2}+x_{2} x_{3}+x_{3} x_{1}\right) \\
= & \left(x_{1}+x_{2}+x_{3}\right)^{2}-2\left(x_{1} x_{2}+x_{2} x_{3}+x_{3} x_{1}\right)-\left(x_{1} x_{2}+x_{2} x_{3}+x_{3} x_{1}\right)
\end{aligned}
$$

The Vieta's formula of cubic equation (102) is

$$
x_{1}+x_{2}+x_{3}=0, x_{1} x_{2}+x_{1} x_{3}+x_{2} x_{3}=p, x_{1} x_{2} x_{3}=-q
$$

According to Equations (145) and (146), there are relations

$$
\begin{gathered}
\alpha^{3}+\beta^{3}=-27 q \\
\alpha \beta=-3 p, \alpha^{3} \beta^{3}=-27 p^{3}
\end{gathered}
$$


So $\alpha^{3}$ and $\beta^{3}$ can be regarded as the roots of the second degree equation with form

$$
y^{2}+27 q y-27 p^{3}=0
$$

By solving Equation (149), we get

$$
\begin{aligned}
\alpha^{3} & =\frac{-27 q+\sqrt{27^{2} q^{2}+4 \times 27 p^{3}}}{2} \\
\beta^{3} & =\frac{-27 q-\sqrt{27^{2} q^{2}+4 \times 27 p^{3}}}{2} \\
\alpha & =3\left[\frac{-q}{2}+\sqrt{\left(\frac{q}{2}\right)^{2}+\left(\frac{p}{3}\right)^{3}}\right]^{\frac{1}{3}} \\
\beta & =3\left[\frac{-q}{2}-\sqrt{\left(\frac{q}{2}\right)^{2}+\left(\frac{p}{3}\right)^{3}}\right]^{\frac{1}{3}}
\end{aligned}
$$

According to the definitions

$$
\begin{gathered}
x_{1}+x_{2}+x_{3}=0 \\
x_{1}+\omega x_{2}+\omega^{2} x_{3}=\alpha \\
x_{1}+\omega x_{3}+\omega^{2} x_{2}=\beta
\end{gathered}
$$

We can obtain the solutions (103)-(105) of cubic equation based on Equations (154)-(156).

\subsection{The Problems Existing in the Galois's Theory to Explain the Resolvent of Cubic Equation}

How to prove the validity of the resolvent of cubic equation by Galois's solvable group theory? The author finds that this is a vague problem in the textbooks and references.

The validity of Galois group on the preliminary solution type is involved here. The permutation group of cubic equation is $S_{3}$. Its unique true subgroup is $A_{3}$ shown in Equation (109), in which the group elements (123) and (132) are even permutation ones. It is generally believed as long as we prove that the solutions $\alpha^{3}$ and $\beta^{3}$ of the cubic equation's resolvent have the invariability of $A_{3}$ group, the validity of Galois's theory is proved. But this is not the true.

It is easy to prove that $\alpha^{3}$ is unchanged under the action of group element (123). We have

$$
\begin{aligned}
(123) \alpha^{3} & =(123)\left(x_{1}+\omega x_{2}+\omega^{2} x_{3}\right)^{3}=\left(x_{2}+\omega x_{3}+\omega^{2} x_{1}\right)^{3} \\
& =\omega^{3}\left(x_{1}+\omega x_{2}+\omega^{2} x_{3}\right)^{3}=\left[\omega\left(x_{2}+\omega x_{3}+\omega^{2} x_{1}\right)\right]^{3} \\
& =\left(\omega x_{2}+\omega^{2} x_{3}+x_{1}\right)^{3}=\alpha^{3}
\end{aligned}
$$

But if the group element (132) is acted on $\alpha^{3}$, the result is 


$$
\begin{aligned}
(132) \alpha^{3} & =\left(x_{3}+\omega x_{1}+\omega^{2} x_{2}\right)^{3}=\omega^{3}\left(x_{3}+\omega x_{1}+\omega^{2} x_{2}\right)^{3} \\
& =\left[\omega\left(x_{3}+\omega x_{1}+\omega^{2} x_{2}\right)\right]^{3}=\left(\omega x_{3}+\omega^{2} x_{1}+x_{2}\right)^{3} \neq \alpha^{3}
\end{aligned}
$$

It is easy to see $(132) \alpha^{3} \neq \beta^{3}$. In fact, the group element (23) is acted on $\alpha^{3}$, we get

$$
\text { (23) } \alpha^{3}=\left(x_{1}+\omega x_{3}+\omega^{2} x_{2}\right)^{3}=\beta^{3}
$$

However, the group element (23) does not belong to $A_{3}$, so the result of Equation (159) just proves the invalidity of Galois solvable groups.

On the other hand, $\alpha^{3}$ and $\beta^{3}$ are not the roots of cubic equation. They are the roots of quadratic equation (149). The roots of cubic equation are $x_{1}, x_{2}, x_{3}$. Using $\alpha^{3}$ and $\beta^{3}$ to replace $x_{1}, x_{2}, x_{3}$ confuses the concepts. According to the Galois's theory of solvable group, the automorphism mapping should be acted on $x_{1}, x_{2}, x_{3}$, rather than acted on $\alpha^{3}$ and $\beta^{3}$. We cannot use the roots of the quadratic equation to construct the Galois group of cubic equation. Although it is valid using them to construct the roots of cubic equations, the validity of Galois' theory cannot be proved based on them.

\subsection{The Resolvent of Quartic Equation}

There were several versions using Galois's theory to construct the resolvent of quartic equation. For example, the versions proposed by Euler and Ferrari, but none of them can obtain the expected results in strict accordance with Galois's theory. The Euler's method is discussed below [14]. The quartic equation is

$$
x^{4}+p x^{2}+q x+c=0
$$

where $x_{1}, x_{2}, x_{3}, x_{4}$ are four roots of Equation (160). Using them to construct three functions below

$$
\begin{aligned}
& \alpha^{2}=\frac{1}{16}\left(x_{1}+x_{3}-x_{2}-x_{4}\right)^{2} \\
& \beta^{2}=\frac{1}{16}\left(x_{2}+x_{3}-x_{1}-x_{4}\right)^{2} \\
& \gamma^{2}=\frac{1}{16}\left(x_{3}+x_{4}-x_{1}-x_{2}\right)^{2}
\end{aligned}
$$

According to Equations (161)-(163) and by considering the Vieta's formula of Equation (160), following relations can be obtained

$$
\begin{aligned}
& \alpha^{2}+\beta^{2}+\gamma^{2} \\
& =\frac{3}{16}\left(x_{1}+x_{2}+x_{3}+x_{4}\right)^{2}-\frac{1}{2}\left(x_{1} x_{2}+x_{1} x_{3}+x_{1} x_{4}+x_{2} x_{3}+x_{2} x_{4}+x_{3} x_{4}\right) \\
& =-\frac{1}{2} p \\
& \quad \alpha^{2} \beta^{2}+\alpha^{2} \gamma^{2}+\beta \gamma^{2} \\
& =\frac{1}{16}\left(x_{1} x_{2}+x_{1} x_{3}+x_{1} x_{4}+x_{2} x_{3}+x_{2} x_{4}+x_{3} x_{4}\right)^{2}-\frac{1}{4} x_{1} x_{2} x_{3} x_{4} \\
& =\frac{1}{16} p^{2}-\frac{1}{4} c
\end{aligned}
$$




$$
\alpha \beta \gamma=\frac{1}{8}\left(x_{1} x_{2} x_{3}+x_{1} x_{2} x_{4}+x_{2} x_{3} x_{4}\right)=-\frac{1}{8} q
$$

Therefore, $\alpha^{2}, \beta^{2}$ and $\gamma^{2}$ are the roots of following cubic equation, called as the resolvent of quartic equation

$$
y^{3}+\frac{1}{2} p y^{2}+\left(\frac{1}{16} p^{2}-\frac{1}{4} c\right) y-\frac{1}{64} q^{2}=0
$$

By solving this equation, three roots are obtained. Take the square root of them, $\alpha, \beta$ and $\gamma$ can be obtained. All of them have two values, so we have four sets of solutions about $\alpha, \beta$ and $\gamma$. Taking any set of them and considering the Vieta formula and Equations (160)-(163), we have

$$
\begin{gathered}
x_{1}+x_{2}+x_{3}+x_{4}=0 \\
\frac{1}{4}\left(x_{1}+x_{3}-x_{2}-x_{4}\right)=\alpha \\
\frac{1}{4}\left(x_{1}+x_{3}-x_{2}-x_{4}\right)=\beta \\
\frac{1}{4}\left(x_{3}+x_{4}-x_{1}-x_{2}\right)=\gamma
\end{gathered}
$$

Based on Equations (167)-(170), a set solution for the quartic equation is obtained with

$$
\begin{aligned}
& x_{1}=\alpha-\beta-\gamma, \quad x_{2}=-\alpha+\beta-\gamma \\
& x_{3}=\alpha+\beta+\gamma, \quad x_{4}=-\alpha-\beta+\gamma
\end{aligned}
$$

\subsection{The Problems Existing in the Galois's Theory to Explain the Resolvent of the Quartic Equation}

Suppose that the first radical solution of Equation (160) is obtained according to the radical extension theory of Galois. The symmetry of equation changes from $S_{4}$ shown in Equation (134) to $A_{4}$ shown in (136). So $\alpha^{2}, \beta^{2}$ and $\gamma^{2}$ should satisfy the invariability of $A_{4}$. However, it is easy to prove that under the replacement of $A_{4}, \alpha^{2}$ can keep unchanged, but $\beta^{2}$ and $\gamma^{2}$ cannot. For example, we have

$$
\begin{aligned}
& (124) \beta^{2}=\frac{1}{16}\left(x_{4}+x_{1}-x_{2}-x_{1}\right)^{2} \neq \beta^{2} \\
& (124) \gamma^{2}=\frac{1}{16}\left(x_{3}+x_{1}-x_{2}-x_{4}\right)^{2} \neq \gamma^{2}
\end{aligned}
$$

On the other hand, if the group elements (12) and (1342) of $S_{4}$ are acted on $\alpha^{2}$, the results are

$$
\begin{aligned}
& (12) \alpha^{2}=\frac{1}{16}\left(x_{2}+x_{3}-x_{1}-x_{4}\right)^{2}=\beta^{2} \\
& (1342) \alpha^{2}=\frac{1}{16}\left(x_{3}+x_{4}-x_{1}-x_{2}\right)^{2}=\gamma^{2}
\end{aligned}
$$


However, as we known that the group elements (12) and (1342) do neither belong to subgroup $A_{4}$ nor belong to subgroup $V$ as shown in Equations (136) and (137).

For the same reason, $\alpha^{2}, \beta^{2}$ and $\gamma^{2}$ are not the roots of quartic equation. They are the roots of cubic equation. The roots of quartic equation are $x_{1}, x_{2}, x_{3}, x_{4}$. Using $\alpha^{2}, \beta^{2}$ and $\gamma^{2}$ to replace $x_{1}, x_{2}, x_{3}, x_{4}$ confuse the concept. According to the Galois's theory of solvable group, the automorphism mapping should be acted on $x_{1}, x_{2}, x_{3}, x_{4}$, rather than acted on $\alpha^{2}, \beta^{2}$ and $\gamma^{2}$. We cannot use the roots of the cubic equation to construct the Galois group of quartic equation. Although it is valid using them to construct the roots of quartic equation, the validity of Galois' theory cannot be proved based on this method.

\section{Conclusions}

Abel and Galois are regarded as the tragic figures in the history of mathematics, who suffered a lot and had a short life. Abel and Galois did not see their theories to be accepted by the world in their lifetime, but in their death they became famous. Their proofs that there were no radical solutions to the quintic equations were regarded as a monument and had never been surpassed.

In order to solve the difficult problems whether the quintic equation had radical solution, based on the previous work, Galois proposed a relatively complete theory called as the group theory, which advanced the traditional algebra to the modern one, made an important contribution to the development of modern mathematics.

Since Abel and Galois's theory was published, the idea that there were no radical solutions to the quanitic and higher degree equations had become the final conclusion. Mainstream mathematicians seemed not to care about this problem, regardless of the Abel and Galois's theory contradicted with the fundamental theorem of algebra. However, the development of science is unexpected. In recent years, the solutions of some special quanitic and sextic degree equations are founded which can be expressed in the radical forms. The theories of Abel and Galois cannot explain why these equations are solvable.

In order to understand the truth of the matter, the author made an in-deep study on Abel and Galois's proof, and found that the situations were completely different from what was expected. The proof of Abel's proof was completely wrong. The basic forms of cubic and quartic equation's solutions were different from what Abel proposed. We have no reason to think that the solutions of higher degree equations would have the forms declared by Abel. Meanwhile, Abel's proof had some primary mistakes, logical confusion and misuse of concepts. All of them made Abel's proof untenable.

Although Galois's group theory was greatly successful, his proof that the quanitic equation had no radical solutions was unsuccessful. Galois's theory was more a conjecture than a proof. And this conjecture was insufficient, far-fetched and untenable, too. 
In fact, Galois's solvable group has no inevitable logic relation with whether a quintic equation is solvable. The symmetric relation between the roots and the coefficients of the quintic equation is described by the permutation group $S_{5}$ which has indicated the existence of the roots of the quintic equation. The fact that the quintic equation has no solvable group only means that there is no symmetric relation of solvable group between the roots and the coefficients of the equation, which does not mean that the equation has no radical solutions.

The concept of automorphism mapping is introduced in order to construct the Galois radical extension group. The relation between the Galois radical extension group and the solvable group is established. According to the original definition, the automorphism operator does not change the root itself, or turns the root into another root of the same equation. However, in order to prove the validity of Galois group for general cubic and quadric equations, the automorphism operators are applied to some relations between the roots, rather than the roots themselves. This directly violates the original definition of the automorphism operator and results in invalid of the proof. Because the relation between the roots can be arbitrary, it results in the missing of uniqueness and universality.

The actual processes to solve the general cubic and quartic equations do not obey the tower structure of Galois solvable group. The Galois's radical expansion theory is inconsistent with the process of solving the equation. To achieve consistency, some intermediate process had to be invented. However, such intermediate process does not exist. Due to the same reason, the Galois's theory of solvable group is also invalid to explain the resolvent of general cubic and quadric equations.

Some progress has been made on the general solution of quintic equation after Abbe and Galois. In 1858, the French mathematician Charles Hermite and the German mathematician Leopold Kronecker independently proved that the general quintic equation could be solved by the elliptic modular functions. In 1870, the French mathematician Marie Ennemond Camillie Jordan proved that any degree polynomial equations could be solved by using such functions [15]. However, the results are not intuitive because these kinds of solutions are represented by infinite series. The summation of infinite series is difficult. It is difficult to derive meaningful results from them. They cannot replace the radical solutions of high degree equations.

Therefore, the conclusion of this paper is that Abel and Galois had not proved that the quintic equation had no radical solutions. Mathematicians should continue to work hard to find the general solutions of quintic and higher degree equations.

\section{Conflicts of Interest}

The author declares no conflicts of interest regarding the publication of this paper. 


\section{References}

[1] Li, W.L. (1998) Mathematical Treasures, a Selection of Historical Documents. Science Press, Beijing, 471-476.

[2] Galois, E. (1846) Discussion on the Process of Pure Analysis. Journal de Mathématiques Pures et Appliqués, 11, 381-444.

[3] Feng, C.T. (2017) From Fundamental Theorem of Algebra to Transcendental Number. Huadong Normal University Press, Shanghai, 17.

[4] Feng, C.T. (2017) From Solving Polynomial Equations to Abel's Impossible Theorem. Huadong Normal University Press, Shanghai, 17.

[5] Tang, J.E. (2012) Several Quintic Equations Which Can Be Solved by Root's Forms. Advanced Mathematics Research, 15, 58-61.

[6] Fan, J. and Kong, Z.H. (2009) Matrix Solutions for Special Six Degree Equation with Real Coefficients. Advanced Mathematics Research, 12, 66-69.

[7] Sheng, X.P. (2002) Matrix Solution for the Real Coefficient Quartic Equations. Mathematics Bulletin, No. 12, 37.

[8] Zheng, L.F. and Shi, Q. (2009) The Solutions of Quintic Equations. National Defense Industry Press, 127.

[9] Cauchy Memoire Ie, nombre des valeurs qu'une fonection peut acquerie. Journal de Pecole polytechnique, 17.

[10] Pesic, P. (2003) Abel's Proof. An Essay on the Sources and Meaning of Mathematical Unsolvability. The MIT Press, Cambridge, 174. https://doi.org/10.7551/mitpress/1043.001.0001

[11] Peng, C.W., Meng, Y., et al. (2018) Study on the Method of Finding Real Roots for Quintic Equations. Examination Journal, Mathematics and Teaching Research, 7, 62.

[12] Feng, C.T. (2018) From the First Degree Equation to the Galois Theory. Huadong Normal University Press, Shanghai, 15, 98, 103.

[13] Xu, C.H. (2018) Aritin Theorem-Classical Mathematical Problems and Galois Theory. Harbin Institute of Technology Press, Harbin, 100, 110.

[14] Zheng, M.Y. (2000) A Brief History of Algebra (IV). Journal of Jingmen Institute of Technology, 15, 89.

[15] Xie, Y.L. (2011) Radical Solution of Algebraic Equation and Galois Theory. Harbin Institute of Technology Press, Harbin, 35-43. 